\title{
Epoch Target Accrual Number Range
}

National Cancer Institute

\section{Source}

National Cancer Institute. Epoch Target Accrual Number Range. NCI Thesaurus. Code C93827.

A range of integers specifying the minimum and maximum number of subjects to be accrued for the epoch. 